\title{
Evaluation of the NINCDS-ADRDA criteria in the differentiation of Alzheimer's disease and frontotemporal dementia
}

\author{
A R Varma, J S Snowden, J J Lloyd, P R Talbot, D M A Mann, D Neary
}

\begin{abstract}
Objectives-The diagnosis of Alzheimer's disease (AD) is now reliant on the use of NINCDS-ADRDA criteria. Other diseases causing dementia are being increasingly recognised-for example, frontotemporal dementia (FTD). Historically, these disorders have not been clearly demarcated from AD. This study assesses the capability of the NINCDS-ADRDA criteria to accurately distinguish AD from FTD in a series of pathologically proved cases.

Methods-The case records of 56 patients (30 with AD, 26 with FTD) who had undergone neuropsychological evaluation, brain imaging, and ultimately postmortem, were assessed in terms of whether at initial diagnosis the NINCDSADRDA criteria were successful in diagnosing those patients who had AD and excluding those who did not.
\end{abstract}

Results-(1) The overall sensitivity of the NINCDS-ADRDA criteria in diagnosing "probable" AD from 56 patients with cortical dementia (AD and FTD) was 0.93. However, the specificity was only 0.23 ; most patients with FTD also fulfilled NINCDS-ADRDA criteria for AD. (2) Cognitive deficits in the realms of orientation and praxis significantly increased the odds of a patient having AD compared with FTD, whereas deficits in problem solving significantly decreased the odds. Neuropsychological impairments in the domains of attention, language, perception, and memory as defined in the NINCDS-ADRDA statement did not contribute to the clinical differentiation of $A D$ and FTD.

Conclusion-NINCDS-ADRDA criteria fail accurately to differentiate AD from FTD. Suggestions to improve the diagnostic specificity of the current criteria are made.

(F Neurol Neurosurg Psychiatry 1999;66:184-188)

Keywords: Alzheimer's disease; frontotemporal dementia; NINCDS-ADRDA criteria

Dr AR Varma, Cerebral

Function Unit, Neurology

Department, Manchester

Royal Infirmary, Oxford

Road, Manchester M13

9WL, UK. Telephone 0044

161276 4138; fax 0044161

2764681 .

Received 28 April 1998 and in revised form

9 July 1998

Accepted 26 August 1998 characterised by profound alteration in personality and social conduct and cognitive impairments predominantly in frontal lobe "executive" functions. ${ }^{12}$ By contrast, in $\mathrm{AD}$ prominent impairments in the domains of memory, visuospatial functions, and language typically occur in the context of well preserved social skills. ${ }^{34}$ Despite striking differences in their neuropsychological profile there is evidence that clinical differentiation remains poor. For example in a study of 21 patients with pathological confirmation of Pick's disease (one of the causes of FTD), $85 \%$ were misdiagnosed during life, their condition misattributed to $\mathrm{AD} .^{5}$ In another pathological study of 170 patients with a clinical diagnosis of $\mathrm{AD}, 12 \%$ of patients were misdiagnosed during life, of whom $19 \%$ were found to have FTD. $^{6}$

The NINCDS-ADRDA criteria ${ }^{7}$ have been widely adopted and relied upon for the diagnosis of $\mathrm{AD}$. It is not known whether the use of these criteria alone would effectively exclude FTD. The purpose of the study was to determine the ability of the criteria to specify at initial presentation "probable" $\mathrm{AD}$ and to exclude non-AD (FTD) from within a group of those two conditions.

\section{Methods}

The case records of 56 consecutive patients with pathological confirmation of either $\mathrm{AD}$ or FTD were analysed. All of these patients had been assessed and reviewed systematically during life in the Cerebral Function Unit, Neurology Department at the Manchester Royal Infirmary as part of a prospective longitudinal study of dementia. The findings of neuropsychological assessments ${ }^{8}$ performed at initial patient presentation were documented by a neurologist (ARV) who was unaware either of the diagnosis or the patient. Information was carefully recorded on standardised forms with respect to (a) mini mental state examination (MMSE) ${ }^{9}$ test scores and $(b)$ the presence or absence of deficits in each of the neuropsychological domains detailed in the NINCDS-ADRDA statement (table 1) - namely, memory, orientation, language, praxis, attention, perception, problem solving, and activities of daily living and social function. In determining impairment, the rater adhered as closely as possible to definitions of impairment given in the criteria statement. Failure on one or more of the specific cognitive tests recommended by the criteria statement to tap a particular cognitive domain was recorded as evidence of impair- 
Table 1 Patients showing deficits in individual cognitive domains specified by NINCDS-ADRDA criteria

\begin{tabular}{lllllll}
\hline Cognitive domain & AD $n(\%)$ & FTD $n(\%)$ & Sensitivity & Specificity & LR & $95 \% C I$ \\
\hline Memory & $28(93.3)$ & $20(76.9)$ & 0.93 & 0.23 & 1.21 & $0.96-1.53$ \\
Orientation & $26(86.6)$ & $12(46.1)$ & 0.87 & 0.54 & 1.88 & $1.21-2.91^{\star}$ \\
Language & $28(93.3)$ & $24(92.3)$ & 0.93 & 0.08 & 1.01 & $0.87-1.17$ \\
Praxis & $28(93.3)$ & $11(42.3)$ & 0.93 & 0.58 & 2.21 & $1.39-3.49^{\star}$ \\
Attention & $24(80)$ & $19(73.1)$ & 0.8 & 0.27 & 1.09 & $0.82-1.47$ \\
Perception & $12(40)$ & $7(26.9)$ & 0.4 & 0.73 & 1.49 & $0.69-3.21$ \\
Problem solving & $12(40)$ & $18(69.2)$ & 0.4 & 0.31 & 0.58 & $0.35-0.96^{\star}$ \\
\hline
\end{tabular}

$\mathrm{LR}=$ positive likelihood ratio for $\mathrm{AD}$; ${ }^{\star} \mathrm{LR}$ significantly different from 1.

ment in that domain. For example, a deficit in language was scored as present if failure occurred on any of the specified language tests including verbal fluency and naming tasks. The scoring was conservative. If there was not well established documented deficit then it was scored absent. Whenever possible the reason for the failure was also detailed.

In addition to the broad domains outlined by the criteria, patients were scored with respect to the more narrowly defined variables of primary language, primary perception, and spatial domains (table 2). A deficit in primary language was defined as impairment in one or more of the linguistic domains of phonology, orthography, morphology, syntax, or lexical semantics. Evidence for impairment was based on the presence of phonemic or semantic paraphasias in conversational speech, repetition or naming tasks, of grammatical errors in spontaneous speech, of impaired syntactic comprehension, or paralexias in reading and spelling errors in writing. Impoverished verbal fluency or word retrieval, in the absence of phonological or semantic errors, was not construed as primary language impairment. The primary language variable was designed to tap impairments attributable to dysfunction in primary cortical language areas and exclude impairments on language tasks secondary to other cognitive deficits. Primary perceptual deficit was defined as a problem in perceptual recognition of objects, line drawings, or faces. Difficulty arising only on complex perceptual tasks such as the Hooper visual test ${ }^{10}$ involving mental integration of visual information would be excluded as difficulties on such complex tasks could arise for reasons outside the realm of primary perception. Patients were designated to have a spatial deficit if they had observable spatial disorientation as evidenced by difficulty in negotiating the environment, orienting clothing when dressing, localisation of objects, or they failed in spatial tasks (tracking a maze, copying hand postures and line drawings, dot location, and dot counting tasks).

Twenty records were analysed by two independent raters (ARV and JSS) to assess interrater reliability. There was good interrater agreement ( $\kappa$ range $0.6-0.8$ for various domains). Missing values were generally low (median 2); this factor is therefore unlikely to affect the interrater reliability scores. The data from a total of 56 case records were available for final analysis. A record was judged to fulfil NINCDS-ADRDA criteria for probable AD if (a) the MMSE score was $\leqslant 24$ and $(b)$ there were demonstrable deficits in memory and at least one other area of cognition as defined by the criteria statement.

\section{STATISTICS}

For the NINCDS-ADRDA criteria and each cognitive domain the sensitivity, specificity, and positive likelihood ratio (LR) (with $95 \%$ confidence intervals) for probable $\mathrm{AD}$ were calculated.

The sensitivity is the true positive fraction, the fraction of subjects with $\mathrm{AD}$ that have a positive test result (have a deficit in that cognitive domain). The specificity is the fraction of subjects that do not have AD and have a negative test result (do not have that particular deficit). The $L^{11}$ for a positive test result (+LR) is defined as the ratio of the fraction of subjects having a positive test result who have the disease to the fraction having a positive test result who do not have the disease. This can be expressed as:

$$
+\mathrm{LR}=\frac{\text { sensitivity }}{1-\text { specificity }}
$$

Sensitivity and specificity are commonly used indices of the usefulness of diagnostic tests. However, they have the drawback that they cannot be easily applied to an individual patient. LRs, by contrast, can be applied to an individual patient and have an easily understood meaning - that is, modifying the pretest odds of disease. An LR of 1 indicates that no information has been provided by the test. A value $>1$ indicates that the test increases the probability of disease prevalence whereas a value $>1$ decreases the probability of disease presence. LRs have the advantage that they relate the post-test odds of a disease to the pretest odds in a simple way; post-test odds $=+\mathrm{LR}$ $\times$ pre-test odds. Odds of a disease is defined as the ratio of the probability of having disease $(\mathrm{P})$ to the probability of lack of disease; odds = $\mathrm{P} /(1-\mathrm{P})$. LRs were considered significant only if they differed from 1 with $95 \%$ confidence.

Table 2 Patients showing deficits in cognitive domains not specified by NINCDS-ADRDA criteria

\begin{tabular}{lllllll}
\hline Cognitive domain & AD $n(\%)$ & FTD $n(\%)$ & Sensitivity & Specificity & LR & $95 \% C I$ \\
\hline Primary language & $22(73)$ & $12(46.1)$ & 0.73 & 0.54 & 1.59 & $1.00-2.54^{\star}$ \\
Primary perception & $9(30)$ & $1(3.8)$ & 0.3 & 0.96 & 7.8 & $1.06-57.53^{\star}$ \\
Spatial & $22(73.3)$ & $2(7.69)$ & 0.73 & 0.92 & 9.53 & $2.47-36.73^{\star}$
\end{tabular}

$\mathrm{LR}=$ positive likelihood ratio for $\mathrm{AD}$; ${ }^{\star} \mathrm{LR}$ significantly different from 1 . 
Table 3 Demographic data

\begin{tabular}{lllll}
\hline Group & Sex (M:F) & $\begin{array}{l}\text { Age of onset } \\
\text { mean years }(S D)\end{array}$ & $\begin{array}{l}\text { Duration of illness } \\
\text { mean years }(S D)\end{array}$ & $\begin{array}{l}\text { MMSE } \\
\text { mean }(S D)\end{array}$ \\
\hline AD & $14: 16$ & $55.7(12.35)$ & $3.29(1.79)$ & $10.69(7.38)$ \\
FTD & $19: 7^{\star}$ & $56.5(9.11)$ & $2.98(1.9)$ & $19.78(6.41)^{\star \star}$ \\
\hline
\end{tabular}

${ }^{\star} \mathrm{p}<0.05 ;{ }^{\star \star} \mathrm{p}<0.000 . \mathrm{M}=$ male; $\mathrm{F}=$ female; $\mathrm{MMSE}=$ mini mental state examination.

Table 4 Patients fulfilling NINCDS-ADRDA criteria for probable AD

\begin{tabular}{lllllll}
\hline $\begin{array}{l}\text { NINCDS-ADRDA } \\
\text { criteria }\end{array}$ & AD n & FTD $n$ & Sensitivity & Specificity & LR & $95 \% C I$ \\
\hline $\begin{array}{l}\text { Positive } \\
\text { Negative }\end{array}$ & 28 & $\begin{array}{l}20 \\
6\end{array}$ & 0.93 & 0.23 & 1.21 & $0.96-1.53$ \\
\hline
\end{tabular}

$\mathrm{LR}=$ positive likelihood ratio for $\mathrm{AD}$.

\section{Results}

Of the 56 patients with pathological confirmation of diagnosis, 30 had AD and 26 FTD. The neuropathological characteristics of these patients have been described previously. ${ }^{12-14}$ The mean age of onset and duration of illness at initial referral were similar in the two groups (table 3). The mean age of the two groups in this study reflect the referral pattern to a neurological unit. The sex distribution was roughly equal in the $\mathrm{AD}$ group whereas males outnumbered females in the FTD group. The mean MMSE score was significantly higher in the FTD group. In the AD group 29 of the 30 patients had an MMSE score $\leqslant 24$ at the time of initial diagnosis; one of these patients had no demonstrable impairment of his memory. Hence, 28 of 30 patients were considered to fulfil NINCDS-ADRDA criteria for probable $\mathrm{AD}$. In the FTD group 21 of the 26 patients had an MMSE score $\leqslant 24$; one of these patients had no demonstrable memory impairment. Hence, 20 of 26 patients were considered to fulfil NINCDS-ADRDA criteria for probable AD. All patients were impaired in their activities of daily living and social function, which had led to their medical referral.

The overall sensitivity of the NINCDSADRDA criteria in diagnosing $\mathrm{AD}$ in this population of patients with cortical dementia (AD or FTD) was 0.93 ; the specificity 0.23 ; the LR 1.21 (table 4). The sensitivity, specificity, and LRs for individual cognitive domains are shown in tables 1 and 2 . The LR is significantly different from 1 if the value of 1 does not lie within the $95 \%$ confidence interval. The five domains for which this was significantly greater than 1 were orientation, praxis, primary language, and spatial and primary perception. For the domain of problem solving the LR was significantly less than 1 . All the 20 patients with FTD who fulfilled NINCDS-ADRDA criteria for probable $\mathrm{AD}$ would also fulfil current Lund-Manchester criteria for FTD. ${ }^{15}$

\section{Discussion}

The NINCDS-ADRDA Work Group was set up to establish and describe clinical criteria for the diagnosis of $\mathrm{AD} .^{7}$ The authors warned that these criteria were not yet fully operational and that the criteria were to be regarded as tentative and subject to change. Fourteen years later these criteria are still widely used in their original form. Patients who fulfil the NINCDSADRDA criteria are accepted as having a clini- cal diagnosis of probable $\mathrm{AD}$, a practice witnessed in most research publications. Undoubtedly these research practices permeate from published literature to clinical practice.

The validation of these criteria at necropsy have been attempted in several studies, with varying results. ${ }^{6}{ }^{16-18}$ The accuracy rate (ratio of the number of pathologically confirmed correct cases of $\mathrm{AD}$ to the number of clinically diagnosed cases of $\mathrm{AD}$, expressed as the percentage of clinical diagnosis of probable AD using NINCDS-ADRDA ranges between 65\% and $92 \%{ }^{6}$ However, these accuracy rates have been derived in all these studies for the diagnosis of pathological $\mathrm{AD}$, which includes $\mathrm{AD}$ alone and mixed cases (AD pathology plus vascular or Lewy bodies or Parkinson's disease pathology or other pathology). The rates fall if they are calculated using $\mathrm{AD}$ alone pathology as the gold standard, and then vary between $56 \%$ and $63 \%$. Needless to say the accuracy rates are even lower for the clinical diagnosis of possible AD. NINCDS-ADRDA criteria are widely applied in the diagnosis of AD. However, the application of these criteria in the differentiation of two cortical dementias, $\mathrm{AD}$ and FTD, has never been studied before.

Both Mendez et $a \bar{l}$ and Litvan et $a l^{19}$ have found that Pick's disease is often underdiagnosed, the clinical misdiagnosis mainly attributable to AD. It is clear, therefore, that FTD is underdiagnosed and that $\mathrm{AD}$ is the commonest misdiagnosis. The diagnostic difficulty is compounded because in both of these disorders the patients are physically well and have few differentiating neurological signs in the early stages of the illness. ${ }^{20}$ The diagnosis mainly depends on a comprehensive neuropsychological evaluation. However, such assessment also requires a careful qualitative analysis of cognition and behaviour because patients can fail quantified psychometric tests for various reasons.

This study shows that the NINCDSADRDA criteria have high sensitivity (0.93), but low specificity (0.23) in the diagnosis of $\mathrm{AD}$ among a group of patients with cortical dementias (AD and FTD) - that is, although most patients with AD fulfil clinical diagnostic criteria for $\mathrm{AD}$, so too do most patients with FTD. Furthermore, whether or not a patient fulfils the criteria does not significantly change the odds of that patient having $\mathrm{AD}$ as opposed to FTD (the LR is not different from 1 with $95 \%$ confidence). These findings suggest that the ability of the criteria to discriminate $\mathrm{AD}$ and FTD is relatively poor. However, this study has also shown that the identification of deficits in certain cognitive domains can contribute to the clinical differentiation of $\mathrm{AD}$ and FTD; deficits in the realm of orientation in time and place and praxis increase the odds of a patient having AD as compared to FTD, whereas deficits in problem solving decrease the odds of a patient having $\mathrm{AD}$ as opposed to FTD (table 1). Deficits in the domains of attention, perception, language, and memory (as broadly defined by the NINCDS-ADRDA statement) do not significantly change the odds of a patient having either condition (table 1). 
Our patient groups (AD and FTD) were similar for their age of onset and duration of illness at presentation (table 3). Thus any differences found in their neuropsychological deficits could not be accounted for by these factors. The patient population is young, reflecting the referral pattern to a neurological centre. FTD is a disorder more commonly seen in the presenium. ${ }^{20}$ The clinical features seen in our patients are indistinguishable from those patients referred to a psychogeriatric centre. ${ }^{1}$ Patients with FTD had a significantly higher MMSE score than the patients with AD (table 3). It is the more striking, given the lesser overall severity of dementia as assessed by this score, that such a high percentage of patients with FTD fulfil criteria for AD (table 4). Failures in individual cognitive domains (tables 1 and 2) across the two study groups (AD and FTD) are discussed below.

Memory impairment is virtually ubiquitous in dementing illnesses. ${ }^{21}$ The NINCDSADRDA criteria do not draw a distinction between memory impairments due to a retention disorder ${ }^{22}$ and those secondary to problems in retrieval and organisation..$^{23}$ The neural substrates and hence the underlying causes for these two types of memory dysfunction are distinct. Patients with $\mathrm{AD}$ have a retention disorder from pathology within their limbic structures, whereas patients with FTD commonly have memory impairment due to retrieval and organisational problems due to frontal lobe deficits. Both groups (AD and FTD) have memory deficit (as witnessed in this study), defined by impairment in memory tests, but failure arises for widely different reasons. These differences can be uncovered by appropriate neuropsychological assessment and would improve the diagnostic specificity of the criteria.

The apparently poor discriminating power of language impairment is probably a reflection of the relatively broad definition of linguistic disorder outlined in the criteria statement. The criteria document suggests a wide range of verbal tasks potentially suitable for eliciting a language deficit, including verbal fluency and the Boston naming test. It is well established that patients with FTD show reduced verbal fluency and naming test performance as part of a general adynamia and impoverishment in motor responses. Indeed, verbal fluency is commonly viewed as a test of frontal lobe executive function. ${ }^{24}$ Such broad criteria for language disturbance will therefore inevitably incorporate patients with FTD. When more narrowly defined criteria for language impairment are adopted based on the presence of impairments in primary linguistic processes then the differentiating value of this cognitive domain increases substantially (tables 1 and 2).

Failure in attention tasks also has poor discriminating value (table 1 ) between $\mathrm{AD}$ and FTD. This is not surprising because deficits in attention are a characteristic feature of FTD. The frontal lobes, primarily involved in FTD are known to have an important role in attention. ${ }^{25}$
The criteria statement suggests various tasks that might be used to detect deficits in visual perception including Gollin incomplete pictures test and the Hooper test. ${ }^{10}$ Patients may fail the Hooper complex perceptual tasks for reasons that are not primarily perceptual but arise for reasons of frontal lobe executive dysfunction such as attentional deficits or failure in the ability to manipulate information mentally. When more stringent criteria were adopted to reflect a primary perceptual impairment, the LR improved fivefold (table 2), although few patients overall showed a deficit in this domain.

The identification of deficits in the realm of praxis significantly increases the odds of a patient having $\mathrm{AD}$ as opposed to FTD. The NINCDS-ADRDA criteria indicate that failure on drawing and block assembly tasks should be considered a disorder of praxis. However, patients may fail these tests due to deficits in praxis or spatial function, or due to poor strategic and organisational skills (frontal deficit). Although it is recognised that spatial abilities are commonly affected in $\mathrm{AD}^{3}$ and strikingly preserved in FTD,,$^{15} 26$ the criteria do not consider spatial function as a separate cognitive domain. Of the $11(42 \%)$ patients with FTD who failed in tests of praxis as defined by the criteria, most failed due to deficits in strategy and organisation; only two $(8 \%)$ patients failed specific spatial tasks (one failed dot counting tasks interpreted as secondary to poor sustained attention; the other did not track a maze due to impaired attention). By contrast $22(73.3 \%)$ patients with $\mathrm{AD}$ had spatial impairment (LR 9.53, table 2), indicating high discriminating value for this domain. The identification of a disorder of spatial function as strictly defined increases considerably the odds of a patient having $\mathrm{AD}$ as opposed to FTD (table 2).

Twenty $(77 \%)$ patients with pathological confirmation of FTD in this study fulfilled NINCDS-ADRDA criteria for AD. Eighteen $(70 \%)$ of these patients fulfilled criteria on the basis of failure in the domains of memory, attention, and problem solving only. In view of the fact that the criteria require deficits in only two cognitive domains (one of which is memory) and the finding that failure in problem solving tasks significantly decreases the odds of a patient having $\mathrm{AD}$ as opposed to FTD, it would seem reasonable to consider the exclusion of problem solving tasks from the criteria.

It has been suggested that formal psychometric testing is rarely necessary for the diagnosis of $\mathrm{AD}$ even though NINCDSADRDA criteria called for confirmation of the dementia by neuropsychological testing. ${ }^{3}$ The findings from the present study suggest, on the contrary, the need for more careful documentation of cognitive impairments. Such an exercise should be aimed at identifying not merely test failure, but in discerning the nature of the cognitive deficits underlying such failure, as this has implications for its neural substrate and for clinical diagnosis. A revision of existing NINCDS-ADRDA criteria involving more 
narrowly defined definitions of cognitive deficits ought to improve diagnostic specificity without loss of overall sensitivity. It is suggested that memory be considered impaired only if the patient is also disoriented in time and place. The amalgamation of memory and orientation into one cognitive domain would improve specificity (table 1). A further characterisation of language and perception as primary language and primary perception will improve specificity too. The addition of spatial function and removal of problem solving and attention should also strengthen the criteria. Because a majority of patients with FTD fulfil the NINCDS-ADRDA criteria it is further suggested that those patients who fulfil the diagnosis of probable $\mathrm{AD}$, but who, in addition, fulfil the published criteria for FTD ${ }^{15}$ should be viewed with diagnostic caution and not confidently considered to have probable AD.

FTD is normally the only non-AD form of dementia that can lead to diagnostic confusion. It has been shown that patients with Lewy body dementia (LBD) may also fulfil NINCDSADRDA criteria for $\mathrm{AD} .{ }^{27}$ The advent of neuroimaging including SPECT, ${ }^{28}$ and better histopathological and immunocytochemical techniques have led to the awareness of different neurodegenerative disorders causing dementia and which have characteristic neuropsychological profiles. When the NINCDSADRDA criteria were first formulated their main aim was to differentiate between $A D$ and vascular dementia. Any future revisions and refinements of the present criteria will need to take into account the diversity of neurodegenerative disorders leading to dementia which have neither the neuropsychological profile nor the characteristic pathology of AD.

We are grateful to the carers of our patients for donating the brains of their relatives for research.

1 Gustafson L. Frontal lobe degeneration of non-Alzheimer type. II. Clinical picture and differential diagnosis. Arch Gerontol Geriatr 1987;6:209-23.

2 Neary D, Snowden JS, Northen B, et al. Dementia of fronta lobe type. F Neurol Neurosurg Psychiatry 1988;51:353-61.

3 Berg L, Morris JC. Diagnosis. In: Terry RD, Katzman R, Bick KL, eds. Alzheimer disease. New York: Raven Press, 1994:9-25.

4 Trimble MR. The dementias. In: Trimble MR. Biological psychiatry. Chichester: John Wiley, 1996:291-324.

5 Mendez MF, Selwood A, Mastri AR, et al. Pick's disease versus Alzheimer's disease: a comparison of clinical characveristics. Neurology 1993;43:289-92.
6 Klatka LA, Schiffer RB, Powers JM, et al. Incorrect diagnosis of Alzheimer's disease, a clinicopathological study. Arch Neurol 1996;53:35-42.

7 McKhann G, Drachman D, Folstein M, et al. Clinical diagnosis of Alzheimer's disease: report of the NINCDSADRDA Work Group under the Auspices of Department of Health and Human Services Task Force on Alzheimer's disease. Neurology 1984;34:939-44

8 Neary D, Snowden JS, Bowen DM, et al. Neuropsychological syndromes in presenile dementia due to cerebral atrophy. F Neurol Neurosurg Psychiatry 1986;49:163-74.

9 Folstein MF, Folstein SE. Mini-mental state: a practical method for grading the cognitive state of patients for the clinician. F Psychiatr Res 1975;12:189-98.

10 Hooper HE. The Hooper visual organisation test. Los Angeles: Western Psychological Services, 1958.

11 Black WC, Armstrong P. Communicating the significance of radiologic test results: the likelihood ratio. American fournal of Radiology 1986;147:1313-8.

12 Mann DMA. The topographical distribution of brain pathology in Alzheimer's disease. Acta Neuropathol (Berl) 1991;83:81-6.

13 Mann DMA, South PW, Snowden JS, et al. Dementia of frontal lobe type; neuropathology and immunohistochemistry. 7 Neurol Neurosurg Psychiatry 1993;56:605-14.

14 Mann DMA, South PW. The topographic distribution of brain atrophy in frontal lobe dementia. Acta Neuropathologica 1993;83:334-40.

15 Brun A, Englund B, Gustafson L, et al. Clinical and neuropathological criteria for frontotemporal dementia. The Lund and Manchester Groups. I Neurol Neurosurg Psychiatry 1994;57:416-8.

16 Galasko D, Hansen LA, Katzman R, et al. Clinicalneuropathological correlations in Alzheimer's disease and related dementias. Arch Neurol 1994;51:888-95.

17 Kukull WA, Larson EB, Reifler BV, et al. The validity of three clinical diagnostic criteria for Alzheimer's disease. Neurology 1990;40:1364-9.

18 Boller F, Lopez OL, Moossy J. Diagnosis of dementia: clinicopathological correlations. Neurology 1989;39:76-9.

19 Litvan I, Agid Y, Sastrj N, et al. What are the obstacles for an accurate clinical diagnosis of Pick's disease? A clinicopathological study. Neurology 1997;49:62-9.

20 Snowden IS, Neary D, Mann DMA. Fronto-temporal dementia. In: Fronto-temporal lobar degeneration: frontodementia. In: Fronto-temporal lobar degeneration: frontoNew York: Churchill Livingstone, 1996:9-41.

21 Snowden JS. Contribution to the differential diagnosis of dementias.1: Neuropsychology. Reviews in Clinical Gerontology 1994;4:227-34.

22 Scoville WB, Milner B. Loss of recent memory after bilateral hippocampal lesions. F Neurol Neurosurg Psychiatry 1957; 20:11-21.

23 Moss MB, Albert MS, Butters N, et al. Differential patterns of memory loss among patients with Alzheimer's disease, Huntington's disease and alcoholic Korsakoff's syndrome. Arch Neurol 1986;43:239-46.

24 Milner B. Some effects of frontal lobectomy in man. In: JM Warren, K Abert, eds. The frontal granular cortex and behaviour. New York: McGraw Hill, 1964:313-31.

25 Stuss DT, Benson FD. Attention. In: Stuss DT, Benson FD, eds. The frontal lobes. New York: Raven Press, 1986:97-106.

26 Barber R, Snowden JS, Craufurd D. Frontotemporal dementia and Alzheimer's disease: retrospective differentiation using information from informants. 7 Neurol Neurosurg Psychiatry 1995;59:61-70.

27 McKeith IG, Fairbairn AF, Perry RF, et al. The clinical diagnosis and misdiagnosis of senile dementia of Lewy body type (SDLT). BrF Psychiatry 1994;165:324-32.

28 Talbot PR, Snowden JS, Lloyd JJ, et al. The contribution of single photon emission tomography to clinical differentiation of degenerative cortical brain disorders. $\mathcal{F}$ Neurol 1995; 242:579-86. 